\title{
Very high-energy muons in neutrino water (ice) telescopes
}

\author{
A.G. Bogdanov', D.V. Evdokimov, S.S. Khokhlov, R.P. Kokoulin, A.A. Petrukhin \\ National Research Nuclear University MEPhI (Moscow Engineering Physics Institute) \\ Kashirskoe shosse, 31, 115409, Moscow, Russian Federation \\ E-mail: agbogdanov@mephi.ru
}

Though the main task of neutrino telescopes with volume about $1 \mathrm{~km}^{3}$ (IceCube, Baikal-GVD, $\mathrm{KM} 3 \mathrm{NeT}$ ) is the search of neutrinos of astrophysical origin, they give a good possibility for investigations of atmospheric muons with energy more than $100 \mathrm{TeV}$ (VHE muons). Measurements of the energy spectrum and angular distribution of such muons give information about primary energy spectrum and mass composition at energies around the knee and also on muon generation processes. In this paper, in order to evaluate the possibilities of studying the spectrum of muons of very high energies by giant underwater (ice) setups, simple analytical calculations of the expected spectra of cascades are carried out. Influence of characteristics of primary cosmic rays and model of interaction on results of muon energy spectrum measurements is discussed.

\section{${ }^{1}$ Speaker}




\section{Introduction}

Muons play an important role in the physics of cosmic rays. The flux of muons is formed as a result of decays of charged mesons (mostly, pions and kaons), which are produced in interactions of primary cosmic ray particles with the nuclei of atmospheric atoms and the subsequent development of nuclear-electromagnetic cascades (extensive air showers, EAS). Therefore, investigations of the energy, spatial, and angular characteristics of the muon component provide important information on both the spectrum and mass composition of primary cosmic radiation and on the characteristics of hadron interactions at high energies. Interest in high-energy muons is also due to a fast progress of neutrino astronomy, since a reliable knowledge of the background fluxes of atmospheric muons and neutrinos is needed for interpretation of the experimental data from large-scale underwater (ice) detectors.

Experiments on detecting cosmic-ray muons can be divided into two groups: measuring the inclusive muon energy spectrum and studying muons in the EAS, where the number of muons is usually measured (or their multiplicity in groups).

At moderate energies the inclusive spectrum of muons in the atmosphere was measured in numerous experiments, which data are in reasonable agreement (10-20\%) with each other and with theoretical calculations. However, at the energies above $10 \mathrm{TeV}$ muon spectrum is poorly studied, the data often contradict each other, and in some experiments an excess of muons is found in comparison with the expected flux for the usual mechanism of their generation as a result of the decays of $\pi, K$-mesons in the atmosphere [1]. The region of muon energies above $100 \mathrm{TeV}$ is of a special interest. Firstly, prompt muons from decays of charmed and other short-lived heavy particles, which are produced as a result of interactions of primary particles with air nuclei, can give appreciable contribution to the muon flux. Secondly, muon energies of the order of hundreds $\mathrm{TeV}$ correspond to $\mathrm{PeV}$ energies of primary cosmic ray particles, where changes in the behavior of various EAS components are observed. Therefore, the knee in the energy spectrum of primary particles, if exists, should lead to a sharp decrease in the muon flux [2]. On the contrary, if the observed changes in the EAS development are connected with inclusion of new physical processes or with the formation of a new state of matter, appearance of an excess of very high energy muons (and neutrinos) is possible [3].

\section{Methods of muon spectrum investigations at energies above $10 \mathrm{TeV}$}

To study the spectrum of muons in the region of the order of $10 \mathrm{TeV}$ and more, the following methods were mainly used: magnetic spectrometer, absorption curve, pair meter and calorimeters.

The most direct method of muon energy spectrum study - magnetic spectrometer technique - did not allow reach energies above $10 \mathrm{TeV}$ because of both technical (the necessity to ensure high magnetic field induction simultaneously with manifold increase of magnetized volume) and physical (increase of probability of secondary electron contamination in the events with the increase of muon energy) reasons.

Method based on the depth-intensity measurements has serious uncertainties in estimation of the surface muon energy related with ambiguities in rock density and its composition, their non-uniformity in depth, and, in case of the mountain overburden, with errors in slant depth evaluation. Besides, this method has a principal upper limitation for accessible muon energies, 
since at depths more than about $10 \mathrm{~km}$ w.e. (in standard rock) the intensity of atmospheric muons becomes lower than the background flux of muons locally produced by neutrinos in the surrounding material. Taking into account energy loss fluctuations, such depth corresponds to effective muon threshold energy about $100 \mathrm{TeV}$.

Pair meter technique [4] of muon energy evaluation is based on measurements of the number and energies of secondary cascades (with $\varepsilon<<E_{\mu}$ ) originated as a result of multiple successive interactions of muon in a thick layer of matter, mainly due to direct electron-positron pair production. At sufficiently high muon energies, in a wide range of relative energy transfers $\varepsilon / E_{\mu} \sim 10^{-3}-10^{-2}$ pair production becomes the dominating muon interaction process, and its cross section rapidly increases with $E_{\mu}$. So, the most important advantage of this method compared to other ones is the possibility to advance into the energy region of at least an order higher magnitude. However, to measure the energies of individual muons with a good accuracy, setups with a large number of detecting layers (of the order of hundreds) and sufficient thickness ( $>500 \mathrm{rad}$. len.) are required.

The method based on calorimetric measurements of the spectrum of electromagnetic cascades induced mainly via muon bremsstrahlung does not have upper physical limit (as well as the pair meter technique). However, possibilities of investigations of muon spectrum at high energies are limited by low probability of the production of bremsstrahlung photons with energies comparable to muon energy $\left(\varepsilon \sim E_{\mu}\right)$, and consequently, by the necessity of the increase of the detector mass in order to compensate rapidly decreasing muon intensity. Further progress in this direction obviously may be ensured by Cherenkov water/ice detectors of giant volume designed for detecting of astrophysical neutrino, which are capable to measure high energy bremsstrahlung cascades caused by interactions of muons. Implementation of this method in the setups of $1 \mathrm{~km}^{3}$ scale (IceCube, Baikal-GVD, KM3NeT) seems not quite difficult.

\section{Different models of the muon energy spectrum}

Two expected features of muon energy spectrum in the PeV region are related with the contribution of prompt muon generation and with the change of the primary cosmic ray spectrum slope. As a reference point for constructing models of the muon energy spectrum, we used T. Gaisser's formula [5], which well describes numerous experimental data on measurements of the muon spectrum in the energy range up to $10 \mathrm{TeV}$ with its extrapolation to the very high energies:

$$
\frac{d N_{\mu}}{d E_{\mu} d \Omega}=0.14 E_{\mu}^{-2.7}\left(\frac{E_{\mu}}{E_{k}}\right)^{-\Delta \gamma}\left(\frac{1}{1+\frac{1.1 E_{\mu} \cos \theta}{115}}+\frac{0.054}{1+\frac{1.1 E_{\mu} \cos \theta}{850}}+R\right)\left[\mathrm{cm}^{-2} \mathrm{~s}^{-1} \mathrm{sr}^{-1} \mathrm{GeV}^{-1}\right] .
$$

The first and the second terms in brackets represent the contributions from $\pi$ - and $K$-decays only. Possible contribution of any fast (in comparison with decays of pions and kaons) processes to generation of muons is usually taken into account by addition of the constant $R$, which is a ratio of the number of prompt muons to the number of charged pions with the same energy at production. Prompt muon generation leads to a flatter muon spectrum and to isotropic angular disribution of high energy muons. Here, we have assumed $R=5 \times 10^{-4}$; this value corresponds to moderately optimistic theoretical predictions, and does not contradict the most of experimental 
upper limits for the prompt muon flux. In this case, differential spectra of prompt muons and usual muons (from pion and kaon decays) cross each other at energy about $300 \mathrm{TeV}$. It is important to note that (in contrast with EAS observation) the inclusive muon spectrum is sensitive mainly to the primary energy per nucleon, thus indicating the knee position in the spectrum of protons. Here, we have assumed that the increase of the power index $\Delta \gamma=0.5$ in differential proton spectrum at $3 \mathrm{PeV}$ is followed by the same steepening of the muon spectrum at 10 times lower muon energy $\left(E_{\mathrm{k}}=0.3 \mathrm{PeV}\right)$. This change of the spectrum shape is taken into account by means of the factor $\left(E_{\mu} / E_{\mathrm{k}}\right)^{-\Delta \gamma}$ in expression (1).

Thus, for further estimates we used different muon energy spectrum models, which are shown in Fig. 1:

1) usual muon spectrum from $\pi$-, $K$-decays in the atmosphere $(\Delta \gamma=0, R=0)$, so, for this model asymptotic differential muon energy spectrum slope is equal to 3.7 ;

2) usual spectrum with the knee at $0.3 \mathrm{PeV}$ in muons and $\Delta \gamma=0.5$;

3) usual spectrum with addition of prompt muons at the level of $R=5 \times 10^{-4}$; in this case, spectrum slope at high energies equals to 2.7 and prompt muons dominate at energies more than about $300 \mathrm{TeV}$ (for a vertical direction);

4) usual spectrum with the knee (model 2) and with addition of prompt muons (model 3).

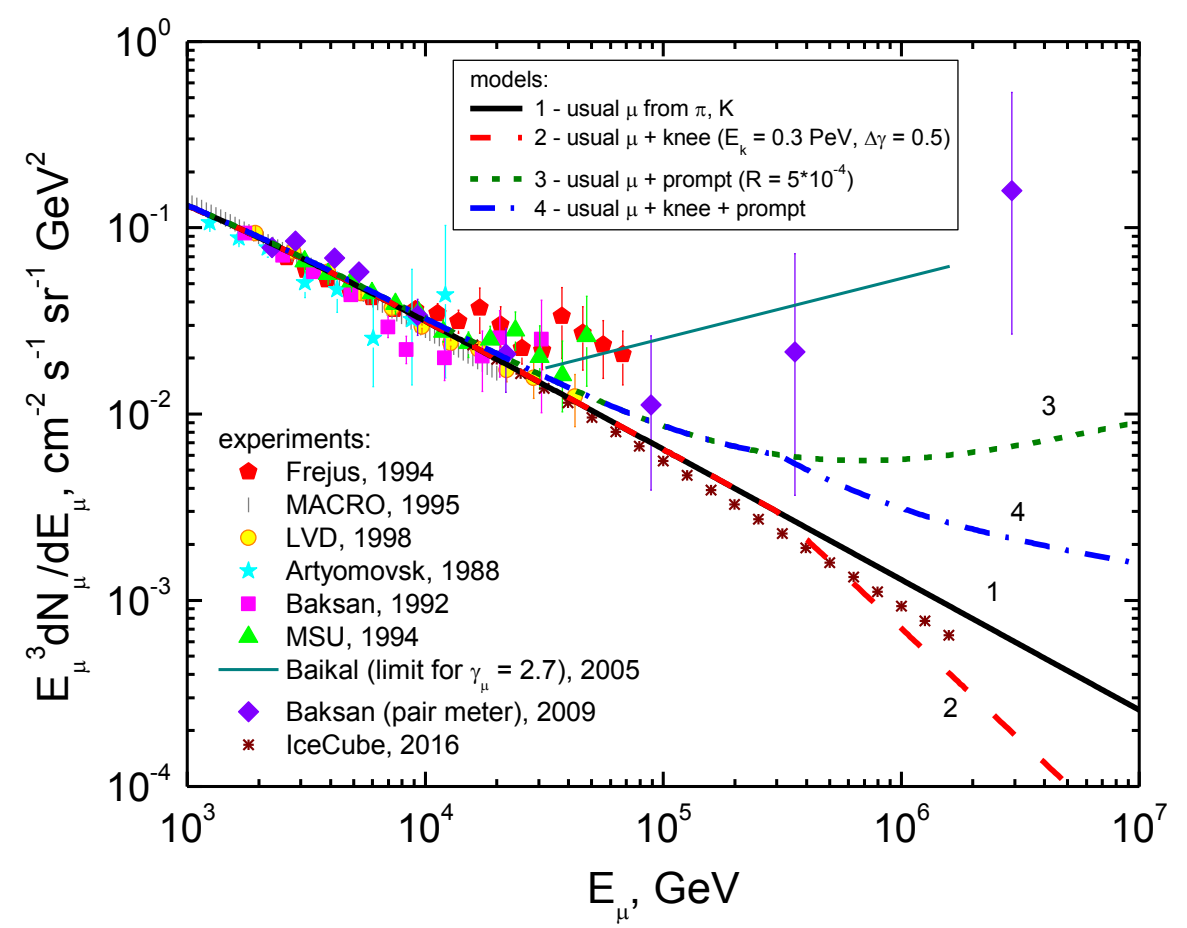

Figure 1: Differential muon energy spectra for vertical direction (experimental data [6-13] and four models).

\section{Underground (underwater) muon energy spectra}

Main features of the underground (underwater) spectrum may be illustrated on the basis of the analytical solution assuming the constancy of coefficients in the muon energy loss relation (and neglecting energy loss fluctuations): 


$$
-d E_{\mu} / d X=a+b E_{\mu},
$$

where $a$ represents the ionization loss and $b$ is the fractional energy loss for radiation processes (both coefficients are slowly varying functions of the energy).

For a power-type differential muon energy spectrum at the Earth's surface:

$$
d N_{\mu} / d E_{\mu}=N_{0} E_{\mu}^{-(\gamma+1)},
$$

one can easily derive the energy spectrum at the depth $h$ :

$$
\frac{d N_{\mu}(h)}{d E_{\mu}}=N_{0} e^{-\gamma b h}\left[E_{\mu}+\frac{a}{b}\left(1-e^{-b h}\right)\right]^{-(\gamma+1)} .
$$

Relation between the energy $E_{\mu}$ of muons at the surface and their energy $E_{\mathrm{h}}$ after passing through thickness $h$ of matter (water, ice, rock) is given by:

$$
E_{\mu}=\left(E_{\mathrm{h}}+a / b\right) e^{b h}-a / b,
$$

besides, the quantity $a / b(\approx 1 \mathrm{TeV}$ in the water) defines a critical energy below which continuous ionization loss is more important than radiative losses.

Energy loss fluctuations due to rare catastrophic collisions with high relative energy transfers, mostly in the processes of muon bremsstrahlung and nuclear interaction, are very important. An accurate correlation between the surface muon spectrum and muon intensity at great depth may be found by a numerical solution of the corresponding transport equation or by Monte Carlo simulation. However, influence of fluctuations may be taken into account by the introduction of some effective energy loss coefficients ( $b_{\text {eff }}$ and $\left.b_{\text {eff }}^{\prime}\right)$ instead of the value $b$ in equation (4) determining the average energy loss rate [14]:

$$
\frac{d N_{\mu}(h)}{d E_{\mu}}=N_{0} e^{-\gamma b_{\text {eff }} h}\left[E_{\mu}+\frac{a}{b_{\text {eff }}^{\prime}}\left(1-e^{-b_{\text {eff }}^{\prime} h}\right)\right]^{-(\gamma+1)},
$$

coefficients $b_{\text {eff }}$ and $b_{\text {eff }}^{\prime}$ slightly depend on the spectrum and on the matter, and $b_{\text {eff }} / b=0.844$ and $b_{\text {eff }}^{\prime} / b=0.752$ for intergal spectrum slope $\gamma=2$.7. Here the coefficients $a$ and $b$ were taken as $2.5 \mathrm{MeV} \mathrm{cm} / \mathrm{g}$ and $3.5 \times 10^{-6} \mathrm{~cm}^{2} / \mathrm{g}$, respectively. The first exponential factor in the expression (6) describes the attenuation of the high energy muon flux with the depths, while the factor in square brackets determines muon spectrum shape at a given depth. The comparison between (4) and (6) shows that the energy loss fluctuations lead to a slower absorption of the muon flux and to an increase of the average muon energy deep underground.

The expected integral muon fluxes on the Earth's surface and at the depth of $2 \mathrm{~km}$ water for the above mentioned (Section 3) muon energy spectrum models are presented in the Fig. 2. One can see that a setup with $1 \mathrm{~km}^{2}$ area may detect for 3 years about 1000 muons with energies above $1 \mathrm{PeV}$ at the surface even for the muon spectrum with the knee. The number of such events can be an order of magnitude larger in the case of the spectrum with prompt muons. However, the number of muons with the same energies at the depth of $2 \mathrm{~km}$ water is almost an order of magnitude less. The influence of energy loss fluctuations on the underwater muon energy spectrum is shown by thin lines; the expected fluxes are a bit higher than for the case when fluctuations are not taken into account. 


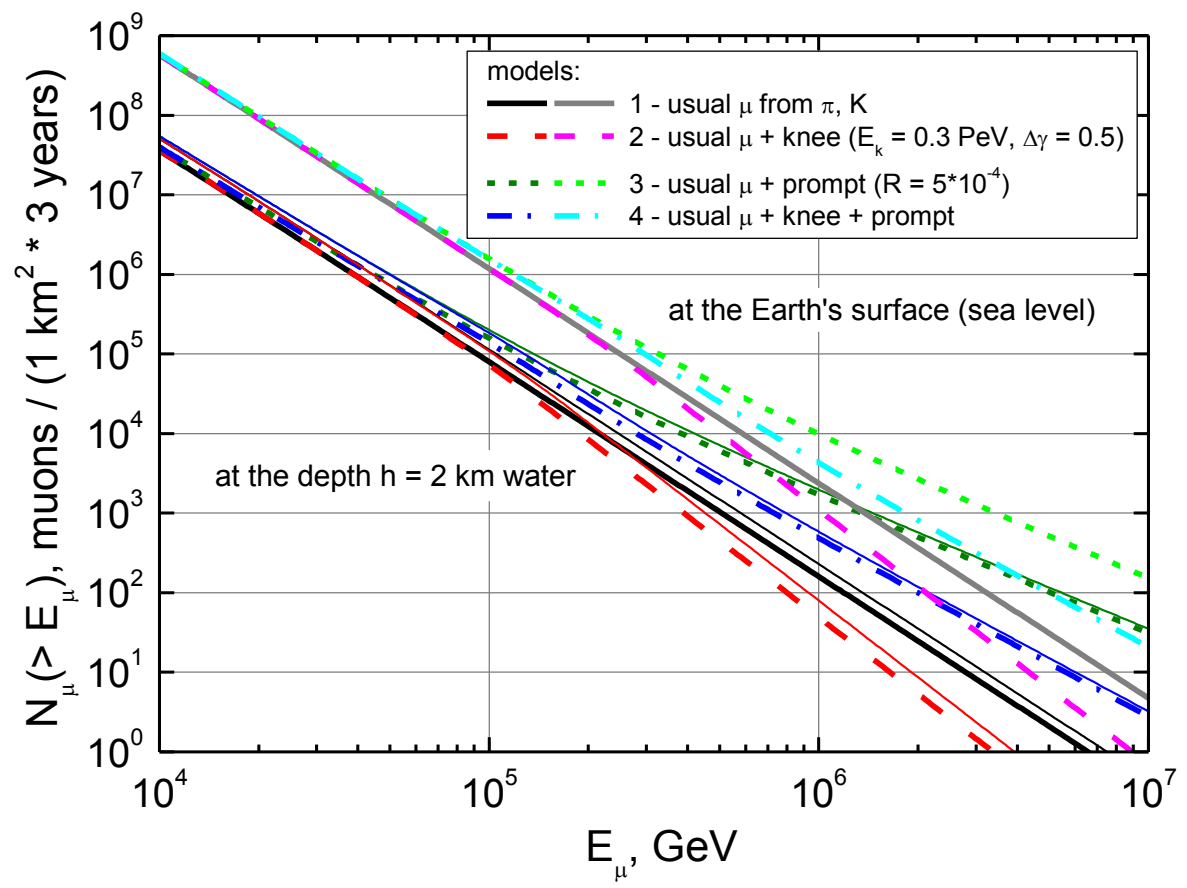

Figure 2: Integral muon energy spectra at the Earth's surface and at the depth of $2 \mathrm{~km}$ water for different models; thin lines show muon spectra subject to the energy loss fluctuations.

\section{Calculations of the muon initiated cascade spectra for large neutrino telescopes}

Simple estimates of the expected number of cascades generated by VHE muons in large underwater (ice) setups can be obtained using analytical expressions without the need for complicated calculations by the Monte Carlo method.

Differential energy spectrum of cascade showers produced via muon interactions in a target with mass $M$ is related to the differential muon spectrum as follows:

$$
\frac{d N_{\mathrm{c}}}{d \varepsilon}=M T \int_{\varepsilon}^{\infty} \sigma(\varepsilon, E) \frac{d N_{\mu}(h)}{d E d \Omega} d E d \Omega,
$$

where $\sigma(\varepsilon, E)$ is the sum of cross sections of electromagnetic muon interaction processes with nuclei (bremsstrahlung [15], electron-positron pair production [16] and inelastic muon scattering [17]), $\varepsilon$ is cascade energy, $\Omega$ and $T$ are solid angle and observation time.

In Fig. 3 the expected (for 3 years data accumulation) integral energy spectra of cascades produced by muons at the depth of $2 \mathrm{~km}$ for underwater setup with a volume of $1 \mathrm{~km}^{3}$ are shown. The calculations have been performed for four different models of the muon spectrum, described in Section 3. It follows from the Fig. 3 that the expected number of cascades with energies more than $100 \mathrm{TeV}$, regardless of the model of the muon spectrum, exceeds 10000 . At that, the estimates of the effective (mean logarithmic) muon energies on the surface are from hundreds $\mathrm{TeV}$ for the near-vertical direction to several $\mathrm{PeV}$ for large zenith angles. Prompt muons should give hundreds of cascades with an energy of more than $1 \mathrm{PeV}$ for 3 years. But, of course, setup acceptance and trigger conditions may limit these statistics several times. 


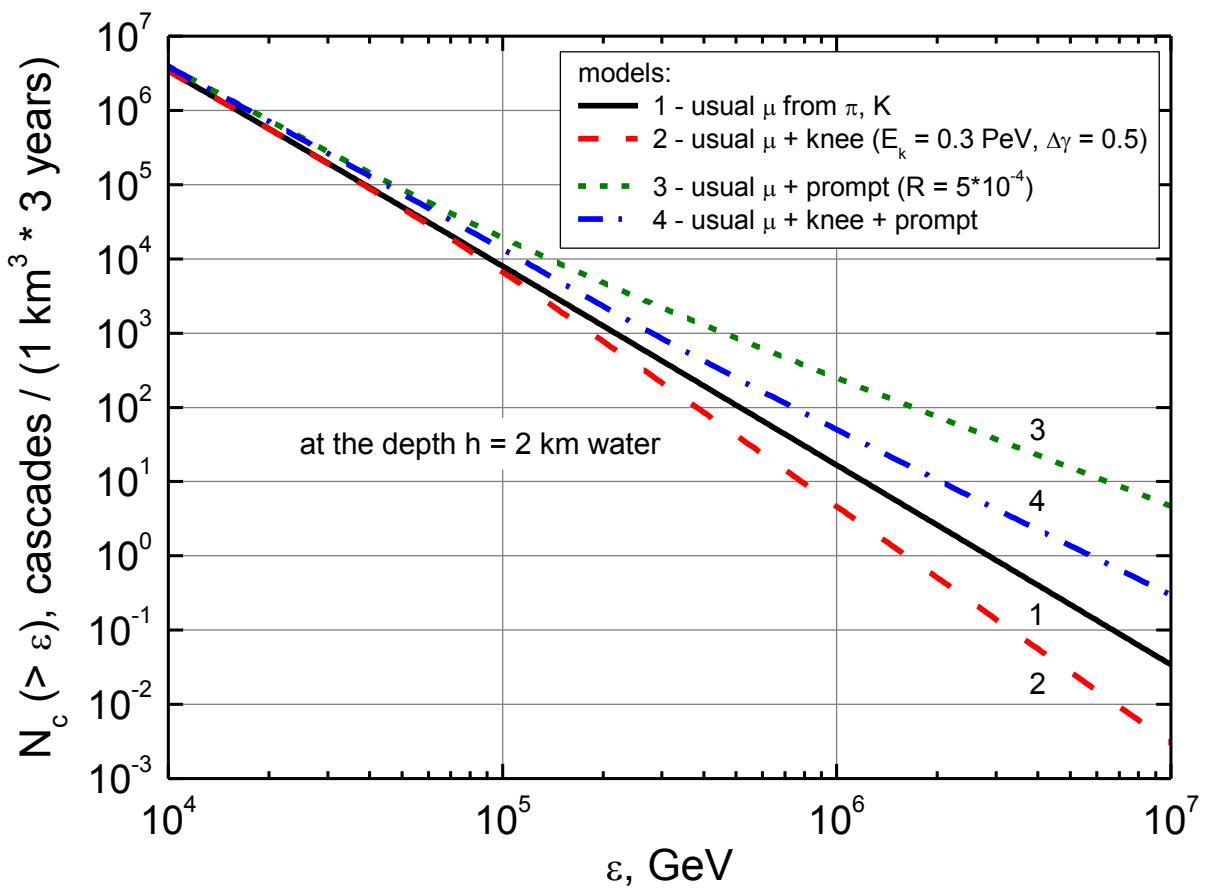

Figure 3: Integral spectra of cascades produced by muons underwater (depth of $2 \mathrm{~km}$ ) for different models of muon energy spectrum.

\section{Conclusions}

Analytical calculations performed in this work give possibility to obtain estimates of the spectrum of cascades generated by muons in giant underwater setups quite simply.

The results of calculations showed that such detectors allow to measure the muon energy spectrum in the PeV energy range, where the changes of muon spectrum connected with prompt muon production and the knee in the primary cosmic ray spectrum should be observed.

The other possibility of high energy spectrum measurements is connected with the use of the pair meter technique - estimation of muon energies via multiple succesive pair production in thick layers of matter. A detailed theoretical analysis of the technique and possibilities of its application for cosmic ray experiments have been published earlier [18].

\section{Acknowledgements}

The work has been performed with the support of the Ministry of Education and Science of the Russian Federation (MEPhI Academic Excellence Project and the government task).

\section{References}

[1] E.V. Bugaev et al., Phys. Rev. D 58 (1998) 05401.

[2] R.P. Kokoulin and I.A. Lyashko, in Proceedings of 2nd International Cosmic Ray Workshop "Aragats 2011", Nor-Amberd, Armenia. Ed. by B. Pattison, R. Martirosov, Armenia, 2011, p. 116.

[3] A.A. Petrukhin, in Proceedings of XIth Rencontres de Blois "Frontiers of Matter", Blois, France, 1999. Ed. by J. Tran Thanh Van, Gioi Publishers, Vietnam, 2001, p. 401. 
[4] I.S. Alekseev, G.T. Zatsepin, in Proceedings of Intern. Conf. on Cosmic Rays, Moscow, Russia, 1960, v. 1, p. 324.

[5] C. Patrignani et al. (Particle Data Group), Chin. Phys. C 40 (2016) 100001.

[6] W. Rhode, Nucl. Phys. B (Proc. Suppl.) 35 (1994) 250.

[7] M. Aglietta et al., Phys. Rev. D 58 (1998) 092005.

[8] V.N. Bakatanov et al., Sov. J. Nucl. Phys. 55 (1992) 1169.

[9] R.I. Enikeev et al., Sov. J. Nucl. Phys. 47 (1988) 665.

[10] G.T. Zatsepin et al., Izv. RAN. Ser. Fiz. 58 N 12 (1994) 119.

[11] V. Aynutdinov et al., Nucl. Instr. and Methods A 602 (2009) 14.

[12] A.G. Bogdanov et al., Astroparticle Physics 36 (2012) 224.

[13] M.G. Aartsen et al., Astroparticle Physics 78 (2016) 1.

[14] R. P. Kokoulin, Nuclear Physics B (Proc. Suppl.) 70 (1999) 475.

[15] S.R. Kelner, R.P. Kokoulin, and A.A. Petrukhin, About cross section for high-energy muon bremsstrahlung, Moscow, Russia, 1995, preprint MEPhI 024-95; CERN SCAN-9 510048.

[16] R.P. Kokoulin and A.A. Petrukhin, Acta Phys. Acad. Sci. Hung., 1970, vol. 29, suppl. 4, p. 277.

[17] V.V. Borog and A.A. Petrukhin, in Proceedings of 14th Intern. Cosmic Ray Conf., Munich, Germany, 1975, vol. 6, p. 1949.

[18] R.P. Kokoulin, A.A. Petrukhin, Nucl. Instr. and Methods A 263 (1988) 468. 